\title{
ÚNG DỤNG MÔ HÌNH KRIGING ĐỂ CHẨN ĐOÁN SỰ CỐ TRONG HỆ THỐNG CHILLER
}

\author{
LÊ TRÀN CẢNH ${ }^{(1)}$, TRÀ̀N ĐÌNH ANH TUẤN ${ }^{(1)}$ \\ AO HÜNG LINH ${ }^{(2)}$ \\ ${ }^{(1)}$ Khoa Công nghệ Nhiệt lạnh, Truờng Đại học Công nghiệp thành phố Hồ Chí Minh; \\ ${ }^{(2)}$ Khoa Công nghệ Cơ khi, Truờng Đại học Công nghiẹp thành phố Hồ Chí Minh; \\ trandinhanhtuan@iuh.edu.vn
}

Tóm tắt. Một mô hình tham số đặc tính chính xác sẽ có vai trò then chốt trong việc nâng cao tỷ lệ chính xác của quá trình phát hiện và chẩn đoán lỗi trong hệ thống chiller. Vì vậy trong nghiên cứu này đã đề xuất sử dụng phương pháp Kriging đóng vai trò là một mô hình tham số để mô hình hóa các đặc tính hoạt động của chiller. Sau đó, kết hợp mô hình này với phương pháp t-test cùng với quy luật chẩn đoán dựa trên độ nhạy cao nhất của tham số đặc tính - để nghiên cứu khảo sát và đánh giá. Bộ dữ liệu thực nghiệm thường được sử dụng hầu hết cho hướng nghiên cứu phát hiện chẩn đoán sự cố trong hệ thống chiller của ASHRAE RP-1043 đã được sử dụng trong nghiên cứu này. Kết quả của nghiên cứu cho thấy rằng, đây là một chiến lược rất thiết thực và có độ chính xác cao.

Từ khóa. Mô hình tham số chiller, Kriging, Phát hiện và chẩn đoán, Chiller ly tâm.

\section{AN APPLICATION OF KRIGING MODELING FOR CHILLER FAULT DETECTION AND DIAGNOSIS STRATEGY}

\begin{abstract}
An accurate parameter model plays an essential role in enhancing the accuracy rate of the chiller fault detection and diagnosis strategy. Thus, Kriging as a parameter model is proposed in this study to capture operating characteristics for a chiller system. Then, a strategy includes the Kriging modeling, $\mathrm{t}-$ test method and a diagnosis rule which is based on the most sensitive feature parameter related fault, is combined in this study. The proposed chiller FDD strategy is tested and validated using the chiller experimental data of ASHRAE RP-1043. Results of this study show that the approximation ability of KRG model achieves high accuracy and this proposed method is robust for fault detection and diagnosis in chiller systems.
\end{abstract}

Keywords. Chiller parameter models, Kriging, Fault detection and diagnosis, Centrifugal chiller.

\section{TỔNG QUAN}

Ngày nay, nhu cầu sử dụng hệ thống máy lạnh chiller trong các trung tâm thương mại, cao ốc... ngày càng tăng. Theo thống kê từ cơ quan năng lượng $\mathrm{Mỹ}(\mathrm{DOE})[1]$ cũng như từ nhiều nghiên cứu khác[2-4] cho thấy rằng, năng lượng tiêu thụ cần thiết cho hệ thống chiller hoạt động sẽ chiếm khoảng 30-40\% tổng năng lượng của một tòa nhà. Khi hệ thống xuất hiện sự cố, sẽ gây tổn thất khoảng 20-30\% nguồn năng lượng này, cũng như chi phí sửa chữa thiết bị hư hỏng do sự cố là khoảng 26\%[5]. Trong thực tế, sự cố hư hỏng là vấn đề không thể tránh khỏi trong quá trình vận hành bất kỳ một hệ thống nào. Sự cố trong hệ thống lạnh cơ bản được chia thành hai dạng là dạng "sư cố cứng" và dạng "sự cố mềm". Sự cố cứng là loại buộc hệ thống phải ngừng hoạt động ngay lập tức khi xuất hiện, ví dụ như cháy động cơ, máy nén bị khựng, hỏng bộ truyền động, hư hỏng thiết bị điện.... Dạng sự cố này thường dễ phát hiện, dễ phân loại được vì xuất hiện một cách đột ngột, khá rõ ràng và kết quả là hệ thống dừng hoạt động ngay lập tức. Do đó không cần thiết phải tiến hành nghiên cứu giải thuật cho các loại sự cố này. Trong khi đó, những sự cố mềm lại diễn biến âm thầm trong một thời gian dài, triệu chứng không hiển thị rõ ràng, như sự tắc nghẽn do tích tụ chất cặn bẩn, dầu trong thiết bị ngưng tụ, thiết bị bay bơi, sự rò rỉ môi chất lạnh, lồi thiết bị cảm biến... dẫn đến cung cấp sai thông tin, vì vậy thường rất khó phát hiện và chẩn đoán ở giai đoạn đầu. Chúng làm suy giảm hiệu suất của hệ thống dần dần, tiêu hao năng lượng tăng dần lên. Chỉ có 
thể phát hiện khi hệ thống đang bị lỗi ở mức độ thực sự nghiêm trọng. Chính vì vậy, việc nghiên cứu tìm giải pháp để có thể giám sát, phát hiện sớm và chẩn đoán sự cố dạng mềm của hệ thống chiller nhằm duy trì hệ thống luôn hoạt động liên tục ở điều kiện tối ưu, qua đó nâng cao hiệu quả sử dụng năng lượng trong tòa nhà, luôn là chủ đề thiết thực mà các nhà khoa học trên thế giới luôn quan tâm trong nhiều thập niên qua. Nhiều nghiên cứu đã chứng minh, nếu có một giải thuật có khả năng phát hiện và chẩn đoán (fault detection and diagnosis-FDD) chính xác sự cố trong hệ thống chiller, sẽ giúp cho người vận hành nâng cao được khả năng đánh giá, dự báo tình trạng hoạt động của hệ thống chiller, đề xuất kịp thời phương án khắc phục, cũng như qua đó tiết kiệm được khoảng 10-40\% năng lượng tiêu thụ của một tòa nhà $[6,7]$.

Nhiều năm qua, phát hiện và chẩn đoán sự cố hư hỏng (FDD) từng được nghiên cứu phát triển trong nhiều lĩnh vực như hàng không, quốc phòng, giám sát điều khiển tự động. Cùng với xu hướng này, việc ứng dụng phương pháp FDD để giám sát điều khiển hệ thống lạnh chiller cũng đã phát triển nhanh chóng trong nhiều thập niên qua. Có thể đề cập đến những phương pháp được sử dụng phổ biến hiện nay như phương pháp phân tích thành phần chính (Principal Component Analysis-PCA)[8], mạng neuron nhân tạo (Artificial Neural Network- ANN)[9], hồi quy tuyến tính (Multiple Linear Regression-MLR)[10]. Nhìn chung, các phương pháp này đã phát triển thực hiện theo các bốn bước chủ yếu sau:

a) Nghiên cứu phát triển mô hình tham chiếu có khả năng mô tả được các đặc tính hoạt động của hệ thống lạnh chiller. Mô hình tham chiếu có nhiệm vụ tiếp nhận và mô hình hóa thông tin dữ liệu từ hệ thống.

b) Xác định ngưỡng hay còn gọi là vùng tin cậy. Nhìn chung, ở bước tính toán này thường sử dụng phương pháp thống kê để xác định ngưỡng theo độ tin cậy như $90 \%, 95 \%, 99 \%, \ldots$ để quan sát và dự báo tình trạng hoạt động của hệ thống. Việc lựa chọn vùng tin cậy là sự cân nhắc cân bằng giữa độ nhạy của phát hiện sự cố và mức độ cảnh báo sai. Vùng tin cậy càng hẹp, khả năng phát hiện sớm sự cố càng tăng lên (nghĩa là: độ nhạy tăng), nhưng đồng thời cũng làm tăng nguy cơ cảnh báo lỗi sai, và ngược lại.

c) Phát hiện sự cố. Giá trị dư, là giá trị chênh lệch giữa giá trị thu thập hay tính toán được so với giá trị ước lượng từ mô hình tham chiếu, được đưa vào vùng tin cậy. Sự cố sẽ được phát hiện nếu giá trị dư nằm ngoài vùng tin cậy đã xác định trước đó.

d) Chẩn đoán sự cố. Tại bước này, kiến thức chuyên gia là yếu tố cốt yếu để xây dựng bảng quy luật lỗi có thể quyết định chính xác hệ thống đang gặp phải sự cố gì.

Có thể thấy rằng, mức độ thành công của một phương pháp FDD phụ thuộc rất nhiều vào độ chính xác của mô hình tham chiếu. Mô hình tham chiếu càng chính xác thì vùng tị cậy càng nhỏ. Vì, theo nguyên lý thống kê, vùng tin cậy được xác định dựa vào hai yếu tố đó là sai số do đo lường (sai số xuất hiện trong quá trình thu thập dữ liệu thông tin) và sai số từ mô hình tham chiếu. Vùng tin cậy càng nhỏ, việc phát hiện sự cố xuất hiện trong hệ thống lạnh chiller càng nhanh chóng hơn. Với lý do đó, bài báo này đề xuất sử dụng Kriging (KRG) để xây dựng mô hình tham chiếu cho hệ thống lạnh chiller. Kriging được gọi là siêu mô hình tham chiếu, từng được sử dụng trong lĩnh vực địa thống kê để nội suy giá trị của một điểm không đo đạc được từ những vị trí đã đo được quanh đó dựa trên phương pháp tính trung bình khoảng cách có trọng số. Tuy nhiên, trong những năm gần đây mô hình KRG được phát triển rất rộng rãi trong nhiều lĩnh vực kỹ thuật khác như ứng dụng KRG để phân tích độ tin cậy[11], dự báo mức độ nhiễm kim loại nặng trong mạch nước ngầm[12], phân tích và dự báo tính ổn định của hệ thống phanh[13], ....và có thể thấy rằng mô hình KRG là một phương pháp rất hứa hẹn cho lĩnh vực xấp xỉ, dự báo các vấn đề trong lĩnh vực kỹ thuật.

\section{DŨ LIỆU THỰC NGHIỆM VÀ PHƯƠNG PHÁP NGHIÊN CÚU}

\subsection{Dũ liệu thực nghiệm}

Số liệu thực nghiệm có ý nghĩa then chốt trong lĩnh vực nghiên cứu phát hiện và chẩn đoán sự cố trong hệ thống chiller. Vì vậy, trong nghiên cứu này đã sử dụng số liệu thực nghiệm từ dự án của ASHRAE RP-1043[14]. Đây là bộ số liệu thực nghiệm được thực hiện với sự tài trợ từ ASHRAE với mục đích nghiên cứu về các sự cố trong hệ thống chiller và từ đó tạo ra một bộ dữ liệu chuẩn cho các nghiên cứu FDD sau này cùng sử dụng. Cho đến hiện nay, vẫn có rất nhiều nhà nghiên cứu trên thế giới trong lĩnh vực này đang tiếp tục sử dụng bộ số liệu này để nghiên cứu phát triển phương pháp mới. Bộ số liệu này đã được tiến hành thực nghiệm thu thập từ hệ thống chiller giải nhiệt nước công suất 90 tấn lạnh. Bộ 
số liệu này bao gồm dữ liệu của chiller hoạt động ở tình trạng bình thường và ở tình trạng có sự cố. Thí nghiệm đã tiến hành mô phỏng và khảo sát bảy loại sự cố "mềm" thường xuất hiện trong hệ thống chiller, thiếu nước cấp thiết bị ngưng tụ $(\operatorname{RedCdW})$, như thiếu nước cấp thiết bị bay hơi (RedEvW), thiết bị ngưng tụ bị bẩn (CdFoul), thừa môi chất lạnh (RefOver), thiếu môi chất lạnh (RefLeak), khí không ngưng $(\mathrm{Ncg})$, thừa dầu bôi trơn (ExOil). Mỗi loại sự cố được tạo ra và giám sát ở bốn mức độ nghiêm trọng tăng dần, nghĩa là $10 \%, 20 \%, 30 \%, 40 \%$ mức độ lỗi. Phương pháp mô phỏng sự cố và mức độ sự cố của bảy loại lỗi này được thống kê trong Bảng 1 .

Bảng 1: Bảy loại sự cố được thực hiện bởi ASHRAE RP-1043

\begin{tabular}{|c|c|c|c|c|c|c|}
\hline \multirow[b]{2}{*}{ Loại sự cố } & \multirow{2}{*}{$\begin{array}{l}\text { Không } \\
\text { có sự cố }\end{array}$} & \multicolumn{4}{|c|}{ Sự cố } & \multirow[b]{2}{*}{ Phương pháp mô phỏng lỗi } \\
\hline & & $\begin{array}{l}\text { Mức } \\
\text { độ } 1\end{array}$ & $\begin{array}{l}\text { Mức } \\
\text { độ } 2\end{array}$ & $\begin{array}{l}\text { Mức } \\
\text { độ } 3\end{array}$ & $\begin{array}{l}\text { Mức } \\
\text { độ } 4\end{array}$ & \\
\hline $\begin{array}{l}\text { Thiếu nước tại } \\
\text { TBNT }\end{array}$ & $61 \mathrm{~m}^{3} / \mathrm{h}$ & $-10 \%$ & $-20 \%$ & $-30 \%$ & $-40 \%$ & Điều tiết lưu lượng nước \\
\hline $\begin{array}{l}\text { Thiếu nước tại } \\
\text { TBBH }\end{array}$ & $49 \mathrm{~m}^{3} / \mathrm{h}$ & $-10 \%$ & $-20 \%$ & $-30 \%$ & $-40 \%$ & Điều tiết lưu lượng nước \\
\hline Tắc TBNT & 164 ống & $-12 \%$ & $-20 \%$ & $-30 \%$ & $-45 \%$ & Cô lập ống theo phần trăm \\
\hline Thừa môi chất lạnh & $136 \mathrm{~kg}$ & $+10 \%$ & $+20 \%$ & $+30 \%$ & $+40 \%$ & $\begin{array}{l}\text { Nạp bổ sung theo tỷ lệ khối } \\
\text { lượng }\end{array}$ \\
\hline Thiếu mối chất lạnh & $136 \mathrm{~kg}$ & $-10 \%$ & $-20 \%$ & $-30 \%$ & $-40 \%$ & Thu hồi theo tỷ lệ khối lượng \\
\hline Khí không ngưng & $0 \%$ & $+1 \%$ & $+2 \%$ & $+3 \%$ & $+5 \%$ & Bổ sung $\mathrm{N}_{2}$ theo tỷ lệ thể tích \\
\hline Thừa dầu bôi trơn & $10 \mathrm{~kg}$ & $+14 \%$ & $+32 \%$ & $+50 \%$ & $+68 \%$ & $\begin{array}{l}\text { Nạp bổ sung theo tỷ lệ khối } \\
\text { lượng. }\end{array}$ \\
\hline
\end{tabular}

Ghi chú: TBNT: thiết bị ngung tụ; TBBH: thiết bị bay hoi.

Theo báo cáo của Comstock và Braun[14], bộ dữ liệu này có tổng cộng ba tập dữ liệu là "Complete data set" có 5191 mẫu, "Reduced data set" có 433 mẫu, và "Steady States" có 27 mẫu. Thông tin của 64 biến tín hiệu trong thí nghiệm này được truy xuất từ hệ thống BMS với thời gian truy xuất dữ liệu định kỳ là 10 giây cho "Complete data set", 2 phút cho "Reduced data set". Trong báo cáo này chỉ sử dụng 02 bộ dữ liệu "Reduced data set" và "Steady States" cho mục đích nghiên cứu.

Vì dữ liệu thô thu thập được luôn tồn tại một phần thông tin nhiễu, nghĩa là thông tin không phản ánh đúng bản chất của hệ thống. Vì vậy để đảm bảo độ chính xác của phương pháp nghiên cứu, bước tiền xử lý dữ liệu đóng vai trò rất quan trọng. Để xử lý thông tin nhiễu, trong báo cáo này tạo vùng cửa sổ lọc với độ dài là 10 giây và độ cao là $3 \sigma$ (độ lệch chuẩn: được xác định theo phương pháp bình phương tối thiểu). Dữ liệu nhiễu sẽ bị loại bỏ khi nằm ngoài vùng cửa sổ lọc này. Kết quả, chỉ còn lại khoảng 30-40\% số mẫu dữ liệu được sử dụng trong nghiên cứu này.

\subsection{Mô hình Kriging (KRG)}

Như đã trình bày ở trên, lý do mô hình KRG được nghiên cứu áp dụng rất rộng rãi trong nhiều lĩnh vực là vì khả năng xấp xỉ và dự báo cho các hệ thống phức tạp có độ chính xác rất cao. KRG có thể giải quyết các vấn đề phi tuyến đa biến bậc thấp lẫn bậc cao trong lĩnh vực kỹ thuật với tốc độ nhanh khi so sánh với các loại mô hình thuật toán khác. Theo tài liệu [15], cấu trúc của mô hình KRG bao gồm hàm số hồi quy toàn cục $\mathrm{g}(\mathrm{x})$ và độ lệch cục bộ $\mathrm{z}(\mathrm{x})$ so với hàm số hồi quy toàn cục, được thể hiện theo công thức (1) sau:

$$
f(x)=g(x)+z(x)
$$

trong đó, $\mathrm{x}$ là ma trận của dữ liệu đầu vào có kích thước tương ứng số biến thiết kế. $\mathrm{G}(\mathrm{x})$ là một mô hình hồi quy đa thức với số bậc có thể là bậc 0 , bậc 1 và bậc 2 . Độ lệch tại mỗi vị trí mẫu được tính toán theo biểu thức (2):

$\operatorname{Cov}\left[\mathrm{z}\left(\mathrm{x}_{\mathrm{i}}\right), \mathrm{z}\left(\mathrm{x}_{\mathrm{j}}\right)\right]=\sigma^{2} \mathrm{R}\left(\theta, \mathrm{x}_{\mathrm{i}}-\mathrm{x}_{\mathrm{j}}\right), \quad \mathrm{i}, \mathrm{j}=1, \ldots, \mathrm{m}$

với $\mathrm{R}($.) có thể được xác định từ một trong các hàm sau hàm mũ, Gaussian, đa tuyến,....[15] Trong đó, hàm Gaussian được sử dụng rộng rãi nhất. Với $\mathrm{m}, \mathrm{n}$ lần lượt là số lượng mẫu và số biến thiết kế, thì hàm số xác định tương quan giữa hai điểm mẫu theo Gaussian được biểu diễn như sau: 
$\mathrm{R}\left(\theta, x_{i}-x_{j}\right)=\exp \left(\sum_{k=1}^{n}-\theta_{k}\left(\mathrm{x}_{k}^{i}-x_{k}^{j}\right)^{2}\right)$

với $\theta \mathrm{k}(0 \leq \theta \mathrm{k} \leq \infty)$ là vector trọng số tương quan tại vị trí thứ $\mathrm{k}$.

\subsection{Phương pháp phát hiện sự cố}

Phát hiện sự cố là bước quan trọng nhằm giúp người vận hành hệ thống có cơ sở để phán đoán tình trạng và đưa ra quyết định hợp lý như "sụ cố vẫn còn chấp nhận", "hệ thống sẽ sủa chũa khi có thể", "chỉ cần điều chỉnh thông số" hay "dùng lập tức để sửa chũa"....Để được như vậy, bên cạnh cần có một mô hình tham chiếu đủ tin cậy, có khả năng mô phỏng chính xác đặc tính hoạt động của hệ thống chiller, thì phương pháp phát hiện sự cố cũng đóng một vai trò rất lớn. Để phát hiện sự cố, cần có một vùng ngưỡng hợp lý. Nếu vùng ngưỡng lớn thì giảm được lỗi cảnh báo sai, nhưng đồng nghĩa với việc làm giảm độ nhạy của phương pháp chẩn đoán và ngược lại. Hiện nay có hai phương pháp xác định vùng ngưỡng thường được sử dụng rộng rãi đó là xác định ngưỡng theo kinh nghiệm người sử dụng và xác định ngưỡng theo phương pháp thống kê. Đối với phương pháp thứ nhất, độ chính xác của việc phát hiện sự cố phụ thuộc hoàn toàn vào kinh nghiệm của người sử dụng vì vậy kết quả thường mang tính chủ quan, cảm tính. Đối với phương pháp thứ hai, nghĩa là phương pháp thống kê, đã và được sử dụng phổ biến nhất hiện nay. Phương pháp này sử dụng giá trị trung bình và độ lệch chuẩn của giá trị dư để xác định vùng ngưỡng ở độ tin cậy nhất định. Giá trị dư được xác định từ việc huấn luyện mô hình tham số bởi tập dữ liệu huấn luyện. Trong nghiên cứu này, sử dụng phương pháp thống kê được đề xuất bởi Cui và Wang [10] để xác định vùng ngưỡng được thể hiện trong công thức sau:

$\tilde{\sigma}_{r_{j}-r_{i}}^{2}=\sum_{j}\left[\left(\frac{\partial g_{i}}{\partial z_{j}}\right) \sigma_{z_{j}}\right]^{2}+\tilde{\sigma}_{Y_{i}}^{2}\left[1+x_{0}^{T}\left(X_{r e g}^{T} X_{r e g}\right)^{-1} x_{0}\right]$

trong đó, $g_{i}$ là công thức tính toán của tham số đặc tính thứ $i . z_{j}$ là biến thứ $j$. $\sigma_{z j}$ là độ lệch chuẩn của $z_{i}$ và $\tilde{\sigma}_{Y i}^{2}$ là phương sai của tham số đặc tính thứ $i$. $x_{0}$ là một ma trận vector cần dự báo hiện hành, $x_{0}^{T}$ là ma trận nghịch đảo của ma trận $\mathrm{x}_{0} . X_{\text {reg }}^{T}$ và $X_{\text {reg }}$ lần lượt là ma trận nghịch đảo và ma trận của tập dữ liệu huấn luyện mô hình tham số.

\subsection{Lựa chọn tham số đặc tính và quy luật chẩn đoán sự cố}

Việc chọn tham số đặc tính đóng vai trò cực kỳ quan trọng trong việc phân loại sự cố. Tham số được chọn cần phải có đầy đủ tính chất vật lý liên quan mật thiết đến sự cố, nghĩa là tham số đặc tính cần phải nhạy với những thay đổi của chế độ làm việc của thiết bị dưới tác động của sự cố có liên quan. Chẳng hạn như, chênh lệch nhiệt độ vào và ra của nước giải nhiệt cho thiết bị ngưng tụ luôn nhạy với sự cố thiếu nước giải nhiệt thiết bị ngưng tụ, nhiệt độ của dầu bôi trơn liên quan đến lượng dầu bôi trơn. Theo nhiều nghiên cứu trước đây[10,16], những sự cố này thường dễ dàng phát hiện và chẩn đoán vì tham số đặc tính liên quan sự cố là duy nhất. Trong khi đó, việc phân loại sự cố tắc thiết bị ngưng tụ, thừa môi chất lạnh và thiếu nước giải nhiệt luôn gặp khó khăn. Bởi vì, khi thiết bị ngưng tụ bị tắc không những làm tăng nhiệt trở mà còn làm giảm lưu lượng giải nhiệt. Kết quả, triệu chứng của ba loại sự cố này là tương tự nhau. Triệu chứng thay đổi của một số tham số đặc tính thường được sử dụng dưới tác động bởi bảy loại sự cố đã được khảo sát và đề xuất nghiên cứu bởi Comstock và Braun được trình bày trong Bảng 2 dưới đây:

Bảng 2: Tín hiệu của tham số đặc tính dưới tác động của sự cố liên quan

\begin{tabular}{|c|c|c|c|c|}
\hline \multirow{2}{*}{ Loại sự cố } & \multicolumn{4}{|c|}{ Tham số đặc tính } \\
\hline & TEI-TEO & TCO-TCI & $\mathrm{T}_{\text {oil }}$ & $\mathrm{LMTD}_{\mathrm{cd}}$ \\
\hline $\begin{array}{l}\text { Thiếu nước tại } \\
\text { TBBH }\end{array}$ & Tăng & Không thay đổi & Không thay đổi & Không thay đổi \\
\hline $\begin{array}{l}\text { Thiếu nước tại } \\
\text { TBNT }\end{array}$ & Không thay đổi & Tăng & Không thay đổi & Tăng \\
\hline Tắc TBNT & Không thay đổi & Tăng & Không thay đổi & Tăng \\
\hline
\end{tabular}




\begin{tabular}{|c|c|c|c|c|}
\hline $\begin{array}{l}\text { Thừa môi chất } \\
\text { lanh }\end{array}$ & Không thay đổi & Tăng & Không thay đổi & Tăng \\
\hline $\begin{array}{l}\text { Thiếu mối chất } \\
\text { lanh }\end{array}$ & Không thay đổi & Không thay đổi & Không thay đổi & Giảm \\
\hline Khí không ngưng & Không thay đổi & Tăng & Không thay đổi & Tăng \\
\hline Thừa dầu bôi trơn & Không thay đổi & Không thay đổi & Tăng & Không thay đổi \\
\hline
\end{tabular}

Trong Bảng 2, TEI-TEO là chênh lệch nhiệt độ nước vào ra tại thiết bị bay hơi, TCO-TCI là chênh lệch nhiệt độ nước vào ra tại thiết bị ngưng tụ, $\mathrm{T}_{\text {oil }}$ là nhiệt độ dầu bôi trơn, $\mathrm{LMTD}_{\mathrm{cd}}$ là chênh lệch nhiệt độ trung bình logarit tại thiết bị ngưng tụ (logarithm mean temperature difference of condenser). Qua đó ta dễ dàng thấy rằng, khi sự cố thiếu nước tại $\mathrm{TBBH}$, thừa dầu bôi trơn và thiếu môi chất lạnh xuất hiện thì chỉ có duy nhất một tham số đặc tính tương ứng với lỗi có sự thay đổi. Ngoài ra, mặc dù có triệu chứng tương tự với các sự cố cố thiếu nước tại TBNT, tắc TBNT và thừa môi chất lạnh, nhưng sự cố khí không ngưng có thể chẩn đoán được khi hệ thống chiller dừng hoạt động[5, 16]. Vì vậy, trong phạm vi nghiên cứu của bài báo này sẽ không thực hiện phân tích chúng. Trái lại, sẽ tiến hành nghiên cứu phân tích các sự cố như tắc TBNT, thiếu nước tại TBNT, thừa môi chất lạnh, vì có xu hướng thay đổi tương tự nhau. Hiện tượng này gây khó khăn trong quá trình chỉ ra lỗi mà hệ thống đang gặp phải.

Để giải quyết vấn đề khó khăn trên, nhóm tác giả sử dụng phương pháp chẩn đoán có tên gọi là "phân tích độ nhạy lớn nhất của tham số đặc tính". Trong phương pháp này dựa vào cả phương hướng thay đổi (tăng, giảm) và mức độ thay đổi của các tham số so sánh với nhau để chỉ ra hệ thống đang xuất hiện sự cố nào. Độ nhạy của tham số đặc tính được xác định bằng công thức (5):

$S_{i}=\frac{R e_{i}}{N g_{i}}$

trong đó, $\mathrm{S}$ (Sensitivity) là giá trị của độ nhạy tham số đặc tính thứ i, $\operatorname{Re}$ ( Residual ) là giá trị dư thứ i, $N g$ là giá trị ngưỡng của tham số đặc tính thứ i. Do sự cố tắc TBNT và thừa môi chất lạnh đều cùng sử dụng $\mathrm{LMTD}_{\mathrm{cd}}$ là tham số đặc tính. Vì vậy để phân loại hai sự cố này, cần phải bổ sung thêm tham số đặc tính $\varepsilon_{\mathrm{sc}}$ là tham số có khuynh hướng khác biệt dưới tác động của hai sự cố đó là tắc TBNT và thừa môi chất lạnh như trong Hình 1 . Tham số đặc tính $\varepsilon_{\mathrm{sc}}$ được xác định bởi biểu thức sau:

$\varepsilon_{s c}=\frac{T_{s u b}}{T_{c d}-T C I}$

trong đó, $T_{s u b}$ là nhiệt độ quá lạnh, $T_{c d}$ là nhiệt độ ngưng tụ, $T C I$ là nhiệt độ nước vào TBNT.

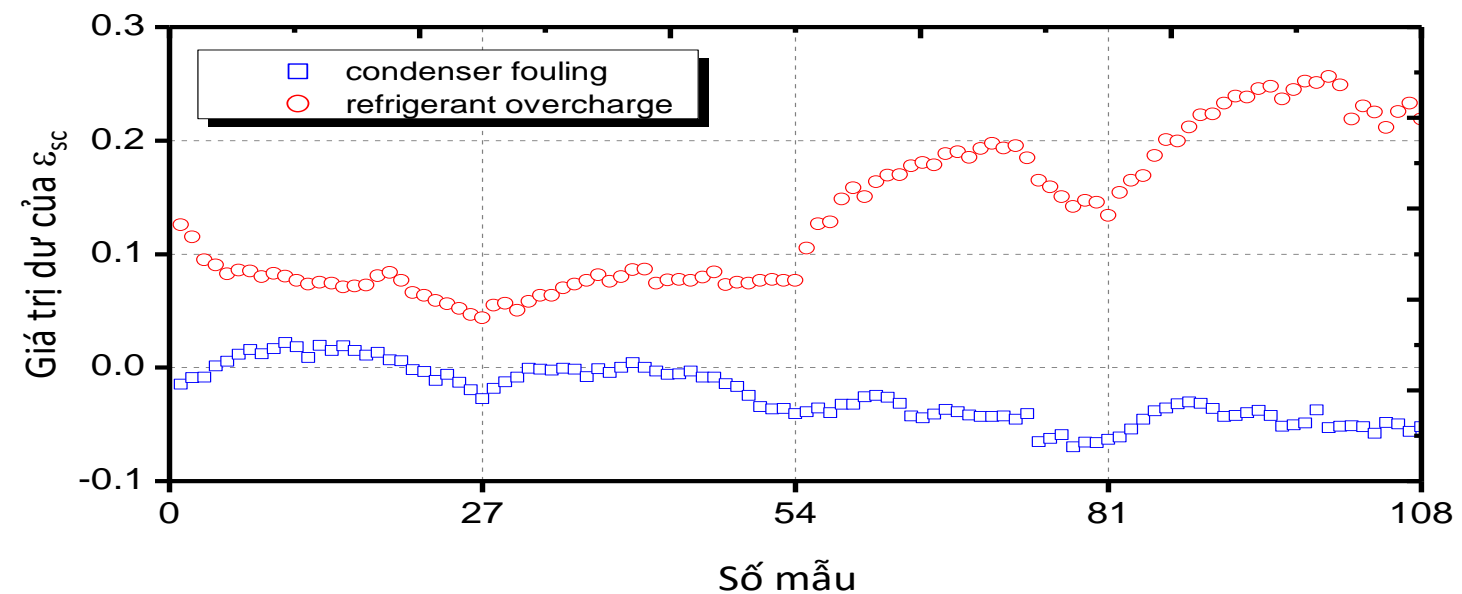

Hình 1: Phương hướng của tham số đặc tính $\varepsilon_{\mathrm{sc}}$ dưới ảnh hưởng của tắc TBNT và thừa môi chất lạnh

Tóm lại, theo phân tích trên, quy luật chẩn đoán được tổng hợp và trình bày trong Bảng 3 .

Bảng 3: Quy luật chẩn đoán sự cố

\begin{tabular}{|c|c|}
\hline Loại sự cố & Quy luật chẩn đoán \\
\hline Thiếu nước tại TBNT & Nếu $\operatorname{Re}_{\text {TCO-TCI }}>\mathrm{Ng}_{\text {TCO-TCI và }} \mathrm{S}_{\mathrm{TCO}-\mathrm{TCI}}>\mathrm{S}_{\mathrm{LMTD} d}$ \\
\hline Tắc TBNT & Nếu $\operatorname{Re}_{\text {LMTDcd }}>\mathrm{Ng}_{\text {LMTDcd }}$ và $S_{\text {LMTDcd }}>S_{\text {TCO-TCI và }} \varepsilon_{\text {sc }}$ giảm \\
\hline Thừa môi chất lạnh & Nếu $\operatorname{Re}_{\text {LMTDcd }}>\mathrm{Ng}_{\text {LMTDcd }}$ và $S_{\text {LMTDcd }}>S_{\text {TCO-TCI và } \varepsilon_{\text {sc }} \text { tăng }}$ \\
\hline
\end{tabular}




\section{KẾT QUẢ VÀ THẢO LUẬN}

\subsection{Huấn luyện mô hình}

Trong nghiên cứu này đã sử dụng bộ số liệu có tên gọi "Normal" của ASHRAE RP-1043 để thực hiện huấn luyện mô hình tham số KRG với ba biến đầu vào là $\mathrm{Q}_{\mathrm{ev}}$ là phụ tải lạnh của hệ thống, TEO là nhiệt độ ra của nước tại TNBH, TCI là nhiệt độ nước vào TBNT. Nghiên cứu đã sử dụng 27 mẫu để thực hiện huấn luyện mô hình KRG. Mô hình KRG sau khi huấn luyện sẽ được kiểm tra lại với số mẫu sủ dụng là 169 mẫu cho mỗi tham số đặc tính (biến đầu ra), cụ thể là LMTD cd $^{2}$ và TCO-TCI. Kết quả được trình bày ở Hình 2.

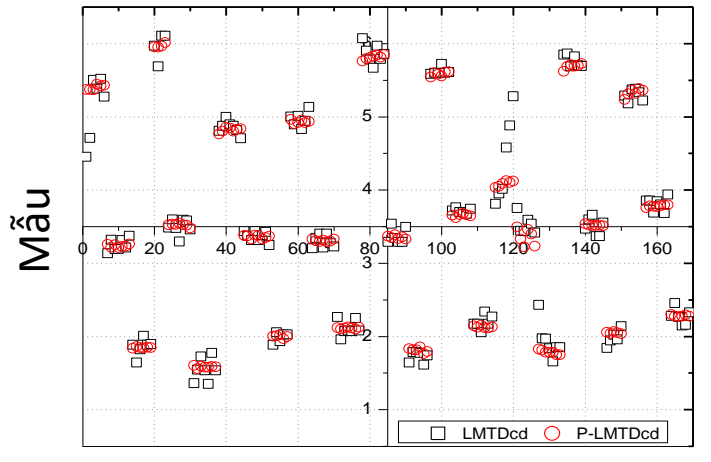

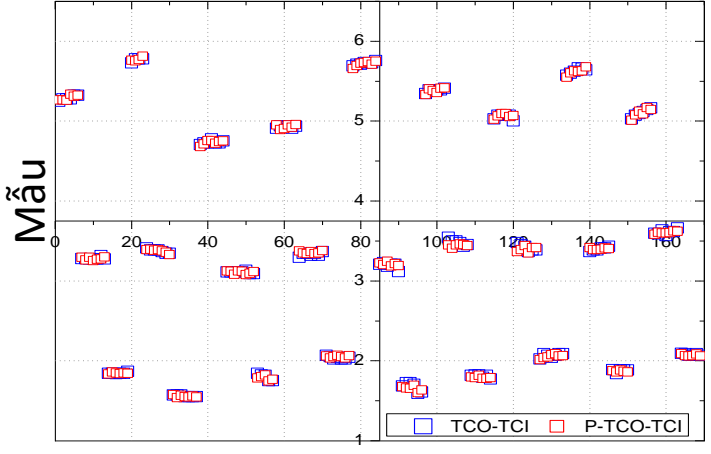

(b)

Hình 2: So sánh giá trị thực nghiệm và giá trị xấp xỉ từ KRG của 02 tham số LMTD $_{\mathrm{cd}}$ và TCO-TCI

Từ Hình 2 dễ dàng thấy rằng, khi so sánh giữa giá trị đo lường được từ bộ dữ liệu thực nghiệm và giá trị xấp xỉ mong đợi đạt được bởi mô hình tham số KRG sau khi huấn luyện có giá trị gần như là tương đồng nhau. Điều này chứng tỏ khả năng xấp xỉ của mô hình tham số KRG có độ chính xác rất cao. Ngoài ra, hệ số tương quan $\mathrm{R}^{2}$ cũng là một trong những thông số thống kê dùng để đo lường mức độ chính xác của một mô hình. Trong nghiên cứu này, giá trị $\mathrm{R}^{2}$ của $\mathrm{LMTD}$ cd và TCO-TCI rất cao, lần lượt là $\mathrm{R}_{\mathrm{LMTDcd}}^{2}=0.9911$ và $\mathrm{R}_{\text {TCO-TCI }}^{2}=0.9998$.

\subsection{Phát hiện và chẩn đoán sự cố}
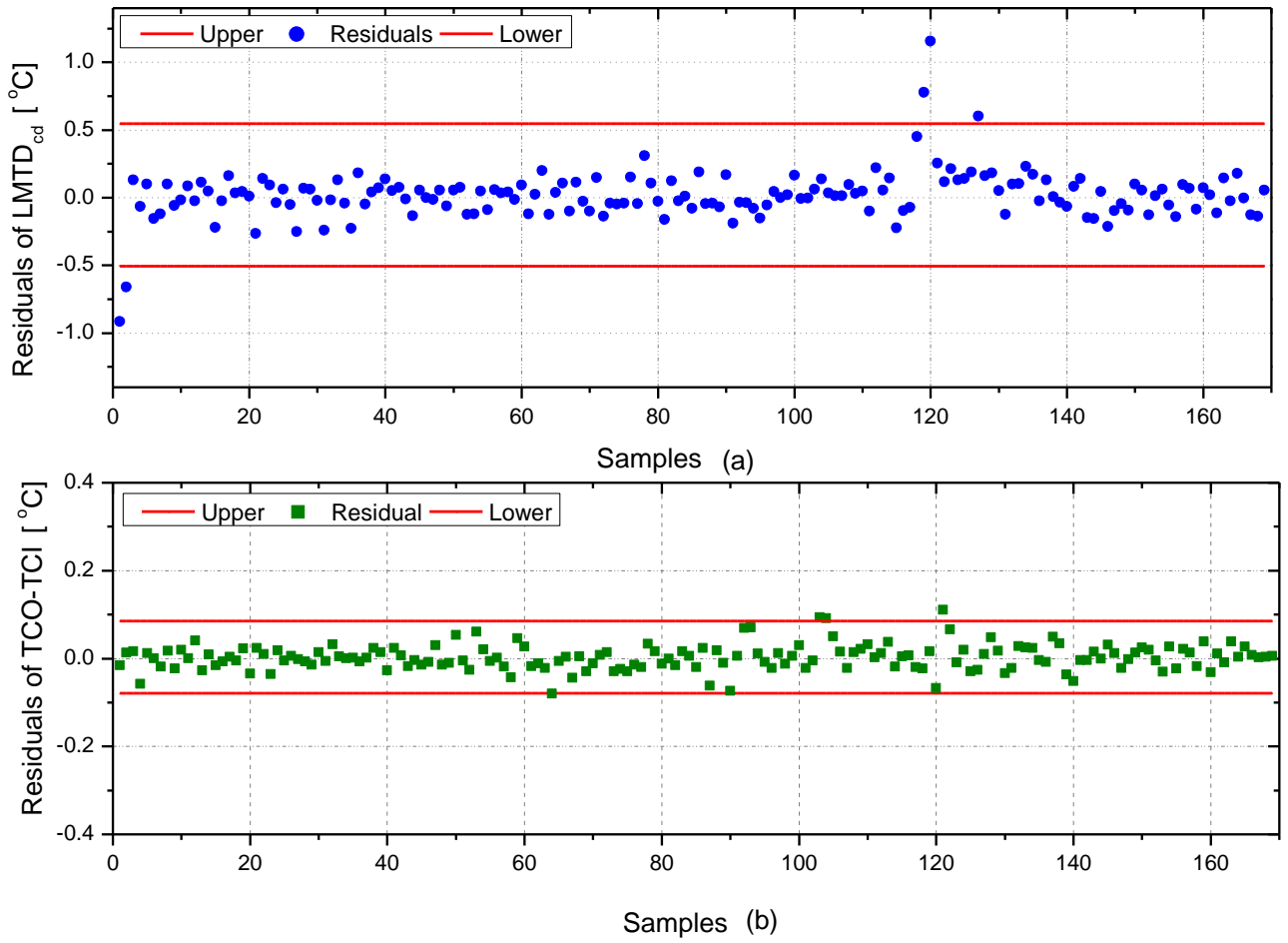

Hình 3: Giá trị dư của tham số đặc tính $\mathrm{LMTD}_{\mathrm{cd}}$ (a) và TCO-TCI (b) ở mức độ $99.73 \%$ độ tin cậy. 

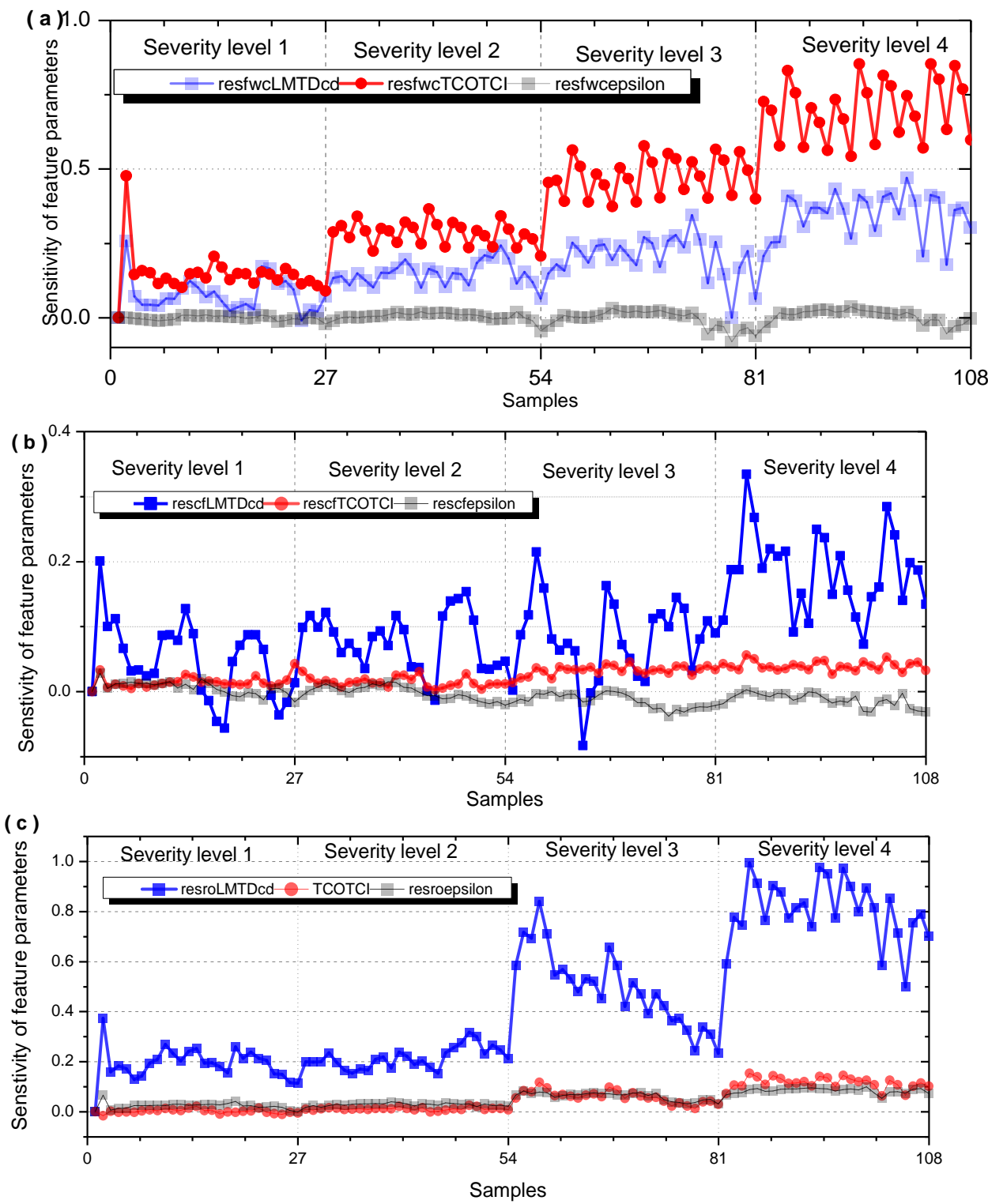

Hình 4: Độ nhạy của các tham số đặc tính dưới tác động bởi sự cố trong chiller.

(a) Thiếu nước giải nhiệt TBNT, (b) Tắc TBNT, (c) Thừa môi chất lạnh.

Bộ số liệu gồm 169 mẫu của "Normal" và 108 mẫu của từng sự cố như thiếu nước tại TBNT, tắc TBNT, thừa môi chất lạnh từ bộ dữ liệu thực nghiệm của ASHRAE-RP1043 được sử dụng để đánh giá khả năng thực hiện phát hiện và chẩn đoán của chiến lược nghiên cứu này. Kết quả được trình bày trong các Hình 3 và Hình 4.

Hình 3 là kết quả của việc thực hiện đánh giá khả năng chẩn đoán của chiến lược nghiên cứu với bộ số liệu thí nghiệm "Normal" mô tả hệ thống chiller đang hoạt động ở trạng thái bình thường. Kết quả cho thấy rằng, chỉ có rất ít điểm dữ liệu của cả hai tham số đặc tính $\mathrm{LMTD}_{\mathrm{cd}}$ và $\mathrm{TCO}-\mathrm{TCI}$ nằm ngoài vùng tin cậy (được xác định với mức $99.73 \%$ độ tin cậy), nghĩa là chỉ có $2.37 \%$ chẩn đoán sai. Kết quả này đồng nghĩa, chiến lược nghiên cứu đã đạt mức độ chẩn đoán chính xác là $97.63 \%$ cho việc xác định hệ thống chiller hiện đang hoạt động ở tình trạng không có sự cố hay không.

Như đã đề cập ở phần trước, trong nghiên cứu này đề xuất chỉ thực hiện chẩn đoán 03 sự cố hư hỏng thường gặp khi vận hành hệ thống chiller đó là thiếu nước giải nhiệt TBNT, tắc TBNT, thừa môi chất lạnh. Trong quá trình kiểm chứng đánh giá, mỗi loại sự cố sẽ có 108 mẫu của bốn mức độ hư hỏng ngày càng tăng, nghĩa là từ mức độ 01 đến mức độ 04 . Hình 04 là kết quả chẩn đoán của chiến lược nghiên cứu. 
Trong Hình 04 , trục tung là kết quả phân tích độ nhạy của từng tham số đặc tính, đó là $\mathrm{LMTD}_{\mathrm{cd}}$, TCO-TCI và $\varepsilon_{\mathrm{sc}}$. Trục hoành là số mẫu được sử dụng để kiểm chứng phương pháp nghiên cứu. Có tổng thể 108 mẫu, mỗi mức độ sự cố là 27 mẫu và mức độ sự cố tăng dần từ trái sang phải của trục hoành. Từ Hình 04 có thể quan sát thây rằng, dưới tác động của 03 loại sự cố này, khuynh hướng của 02 tham số đặc tính $\mathrm{LMTD}_{\mathrm{cd}}$ và TCO-TCI đều tăng. Mức độ tăng ngày càng cao tùy thuộc vào mức độ nghiêm trọng gây ra bởi sự cố. Ở Hình 4.a, dưới ảnh hưởng của sự cố thiếu nước tại TBNT, độ nhạy của TCO-TCI hầu như lớn hơn độ nhạy của $\mathrm{LMTD}_{\mathrm{cd}}$ tại các mức độ nghiêm trọng của sự cố. Điều này chứng tỏ thiếu nước giải

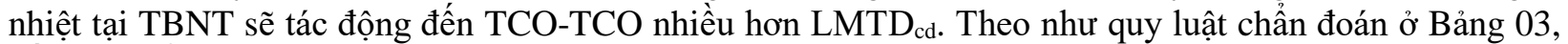
kết quả chẩn đoán chính xác đạt được là $98.15 \%$. Tương tự, Hình 4.b\&c mô tả thông tin độ nhạy của 03 tham số đặc tính dưới tác động của sự cố TBNT bị tắc và thừa môi chất lạnh. Rõ ràng thấy rằng, ở hai sự cố này, cả $\mathrm{LMTD}_{\mathrm{cd}}$ và TCO-TCI đều có khuynh hướng giống nhau, nghĩa là tăng dần theo mức độ nghiêm trọng của sự cố và độ nhạy của $\mathrm{LMTD}_{\text {cd }}$ đa phần lớn hơn TCO-TCI. Vì vậy để phân loại hai sự cố này, buộc chúng ta phải sử dụng thêm tín hiệu của $\varepsilon_{\mathrm{sc}}$. Kết quả chẩn đoán chính xác đạt $20.37 \%$ đối với sự cố tắc TBNT, $71.29 \%$ đối với sự cố thừa môi chất lạnh.

Qua kết quả cho thấy rằng, việc phát hiện và chẩn đoán sự cố thiếu nước giải nhiệt cho TBNT là dễ dàng nhất, có thể sớm phát hiện khi sự cố vừa chớm. Riêng đối với sự cố tắc TBNT là khó chẩn đoán nhất, chỉ có thể phát hiện và chẩn đoán khi mức độ sự cố thực sự đã nghiêm trọng, tức là ở giai đoạn 03 và 04.

\section{KẾT LUẬN}

Nghiên cứu này đã đề xuất một phương pháp nghiên cứu thiết thực cho việc thực hiện phát hiện và chẩn đoán. Trong đó KRG với vai trò là một mô hình tham số đã chứng minh khả năng nắm bắt rất chính xác những đặc tính hoạt động của hệ thống chiller. Phương pháp đã thể hiện được khả năng phát hiện và chẩn đoán ba sự cố tiêu biểu, thường gặp trong chiller với độ chính xác cao. Cụ thể, đạt 97.63\%,98.15\% và $71.29 \%$ độ chính xác cho lần lượt xác định hệ thống chiller đang hoạt động bình thường, thiếu nước giải nhiệt TBNT và thừa môi chất lạnh ở mức $99.73 \%$ độ tin cậy.

Tuy nhiên, trong nghiên cứu này còn việc chẩn đoán sự cố tắc TBNT chưa có độ chính xác cao, cụ thể chỉ có $20.37 \%$ độ chính xác. Và, sự cố này chỉ có thể chẩn đoán được khi sự cố đã thực sự nghiêm trọng, nghĩa là ở mức 03 và 04 . Vì vậy đề xuất cần tiếp tục cải thiện trong các nghiên cứu sau này.

\section{TÀI LIỆU THAM KHẢO}

[1] US department of Energy, Buildings energy data book, 2009.

[2] L Jayamaha, Energy effficiency building systems: green strategies for operation and maintenance, 7th ed. McGraw-Hill, 2007.

[3] R Saidur, M Hasanuzzaman, T M I Mahlia, N A Rahim, H A Mohammed, Chillers energy consumption, energy savings and emission analysis in an institutional buildings, Energy, 36 (8) (2011) 5233-5238.

[4] Z Ma, S Wang, Building energy research in Hong Kong: A review, Renewable and Sustainable Energy Reviews, 13 (8) (2009) 1870-1883.

[5] Y Zhao, S Wang, F Xiao, A statistical fault detection and diagnosis method for centrifugal chillers based on exponentially-weighted moving average control charts and support vector regression, Applied Thermal Engineering, 51 (1-2) (2013) 560-572.

[6] H Wang, Y Chen, C W H Chan, J Qin, J Wang, Online model-based fault detection and diagnosis strategy for VAV air handling units, Energy and Buildings, 55 (0) (2012) 252-263.

[7] C GH, Building control systems, in Oxford: Butterworth-Heinemann, 2000.

[8] Y Hu, H Chen, J Xie, X Yang, C Zhou, Chiller sensor fault detection using a self-Adaptive Principal Component Analysis method, Energy and Buildings, 54 (0) (2012) 252-258. 
[9] N Kocyigit, Fault and sensor error diagnostic strategies for a vapor compression refrigeration system by using fuzzy inference systems and artificial neural network, International Journal of Refrigeration, 50 (2015) 69-79.

[10] J Cui, S Wang, A model-based online fault detection and diagnosis strategy for centrifugal chiller systems, International Journal of Thermal Sciences, 44 (10) (2005) 986-999.

[11] Z Sun, J Wang, R Li, C Tong, LIF: A new Kriging based learning function and its application to structural reliability analysis, Reliability Engineering \& System Safety, 157 (2017) 152-165.

[12] L Belkhiri, L Mouni, T Sheikhy Narany, A Tiri, Evaluation of potential health risk of heavy metals in groundwater using the integration of indicator kriging and multivariate statistical methods, Groundwater for Sustainable Development, 4 (2017) 12-22.

[13] L Nechak, F Gillot, S Besset, J J Sinou, Sensitivity analysis and Kriging based models for robust stability analysis of brake systems, Mechanics Research Communications, 69 (2015) 136-145.

[14] M Comstock, J Braun, Fault detection and diagnostic (FDD) requirements and evaluation tools for chillers, West Lafayette, IN: ASHRAE, (2002).

[15] S N.Lophaven, H B Nielsen, J Sondergraard, DACE-A Matlab Kriging Toolbox, Technical University of Denmark, 2002.

[16] F Xiao, C Zheng, S Wang, A fault detection and diagnosis strategy with enhanced sensitivity for centrifugal chillers, Applied Thermal Engineering, 31 (17-18) (2011) 3963-3970.

Ngày nhận bài: 01/04/2017

Ngày chấp nhận đăng: 20/05/2017 